\title{
Sp1, but not Sp3, functions to mediate promoter activation by TGF- $\beta$ through canonical Sp1 binding sites
}

\author{
Jian-Ming Li, Michael B. Datto, Xing Shen, Patrick Pei-Chih Hu, Yong Yu and \\ Xiao-Fan Wang*
}

Department of Pharmacology and Cancer Biology, Duke University Medical Center, Durham, NC 27708, USA

Received January 6, 1998; Revised and Accepted April 3, 1998

\begin{abstract}
Transforming growth factor $\beta$ (TGF- $\beta$ ) causes growth arrest at the G1 phase of the cell cycle in most cell types. Both the cyclin dependent kinase inhibitor p15 INK4B and p21(Cip1/WAF1) genes have been found to be induced by TGF- $\beta$ in human keratinocyte $\mathrm{HaCaT}$ cells. Analyses of the human p15 and p21 promoters have led to the identification of GC-rich sequences capable of binding to Sp1 transcription factors as necessary elements for the TGF- $\beta$ induction of both promoters. We report here that canonical Sp1 binding sites derived from the SV40 21 bp repeat could also support promoter induction by TGF- $\beta$ when placed upstream of a minimal luciferase reporter construct containing only the TATA and Inr elements. Gel retardation assays identified $\mathrm{Sp} 1, \mathrm{Sp} 3$ and $\Delta \mathrm{Sp} 3$ as major factors binding to the canonical Sp1 sites in $\mathrm{HaCaT}$ cells and that TGF- $\beta$ treatment did not change their binding activities over a $24 \mathrm{~h}$ period. More importantly, GAL4-Sp1, but not GAL4-Sp3, chimeric protein supported TGF- $\beta$ mediated gene induction from a luciferase reporter construct driven by five GAL4 DNA binding sites. Our results suggest that Sp1 binding site can function as a distinct TGF- $\beta$ responsive element for TGF- $\beta$ mediated promoter expression and Sp1 per se can mediate this response.
\end{abstract}

\section{INTRODUCTION}

Transforming growth factor $\beta$ (TGF- $\beta$ ) is a multifunctional cytokine involved in the regulation of many biological processes such as cell proliferation, differentiation, morphogenesis and apoptosis in diverse cell types (1-3). It causes reversible growth inhibition in most lymphoid and epithelial cells such as human keratinocyte $\mathrm{HaCaT}$ cells. In HaCaT cells, TGF- $\beta$ treatment causes rapid induction of the cyclin dependent kinase inhibitors p15 INK4B and p21(Cip1/WAF1) $(4,5)$, which in turn inhibit the enzymatic activities of CDK4/6-cyclin D and CDK2-cyclin E complexes responsible for the phosphorylation of the retinoblastoma protein $(\mathrm{RB})$. Hypophosphorylated RB binds to transcription factors, including the E2F family of transcription factors, needed for $\mathrm{G} 1$ to $\mathrm{S}$ phase progression of the cell cycle, therefore leading to cell cycle arrest at late phase of G1 (6).

Previously, we have demonstrated that a minimal $113 \mathrm{bp}$ promoter sequence of the human 15 gene, containing an Sp1 consensus site capable of binding to the $\mathrm{Sp} 1$ family of transcription factors, was sufficient to support TGF- $\beta$ mediated promoter activation (7). We have also demonstrated an $\mathrm{Sp} 1$ binding site to be required for TGF- $\beta$ induction from the $\mathrm{p} 21$ (Cip1/WAF1) promoter in $\mathrm{HaCaT}$ cells (8). Those results suggest that specific Sp1 binding sites can possibly function as TGF- $\beta$ responsive elements. It is not known, however, whether the Sp1 binding sites from the p15 and p21 promoters represent a distinct class of Sp1 binding sites for TGF- $\beta$ mediated promoter induction, or more generic, canonical Sp1 binding sites can function as TGF- $\beta$ responsive elements. Furthermore, it is not known if specific transcription factors capable of binding to the Sp1 sites mediate the TGF- $\beta$ transactivating signal.

We report here that Sp1 consensus sites from SV40 enhancer of the early promoter can support TGF- $\beta$ mediated promoter activation, suggesting that canonical $\mathrm{Sp} 1$ sites can function as TGF- $\beta$ responsive elements for gene activation. More importantly, GAL4-Sp1, but not GAL4-Sp3, chimeric protein could support TGF- $\beta$ induction from a minimal promoter driven by five GAL4 DNA binding sites, supporting the notion that $\mathrm{Sp} 1$ is the mediator of the TGF- $\beta$ signal.

\section{MATERIALS AND METHODS}

Materials and plasmids

Human TGF- $\beta 1$ was a generous gift from Amgen, Inc. pCAL2 reporter construct was a generous gift from Dr Derynck (UCSF) (9). PAI-1 luciferase reporter was a generous gift from Dr Loskutoff (Scripps Clinic) (10). T $\beta \mathrm{RI}_{\mathrm{KR}}$ and $\mathrm{T} \beta \mathrm{RII}_{\mathrm{KR}}$ have been described previously (11). TI-luc, which contains the TATA box and Inr element in front of the pGL2 Basic luciferase reporter plasmid, was a generous gift from Dr Smale (UCLA) (12). TI+Spp15-luc,

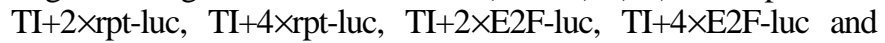
$\mathrm{TI}+5 \times$ Gal4-luc were respectively created by inserting the appropriate oligonucleotides into the SmaI restriction site upstream 
of the TATA box present on the TI-luc. These oligonucleotides have the following sequences: $\mathrm{Sp}^{\mathrm{p} 15}$, AGG GGG CGG AGC; rpt, 5'-TGG GCG GAG TTA GGG GCG GGA-3'; E2F, 5'-ACA ATT TCG CGC CAA ACT TG-3'; GAL4, 5'-CGG AGG ACT GTC CTC CG-3'. Rabbit anti-human Sp1 (sc059), Sp3 (sc644) and c-Myc (sc042) antibodies were purchased from Santa Cruz Biotechnology.

The full length p21 promoter luciferase reporter construct, $\mathrm{p} 21 \mathrm{P}$, as well as the minimal TATA box-Initiator sequence reporter construct, pGL2 T+I have been previously described (8). The GAL4 DNA binding domain expression construct pGAL0, the GAL4 DNA binding domain fusion expression constructs, pGAL4-Sp1-B, pGAL4-Sp1-BQ, pGAL4-Sp1-AQ and pGAL4Vp16, the GAL4 reporter construct, $5 \times$ GAL4-CAT, and the Sp3 expression construct CMV-Sp3-Flu have also been described previously (13). The expression construct pGAL4-Sp1-Bc and pGAL4-Sp1-A were the generous gifts of Dr R.Tjian. The luciferase reporter construct, pGL2 T+I $5 \times$ GAL4, was created by cloning a blunt-ended XbaI-Pst I fragment containing five concatmerized GAL4 DNA binding sites (CGGAGTACTGTACTCCG), from the vector 5×GAL4-CAT into the EcoRV site of pGL2 T+I. The GAL4-Sp3 fusion constructs GAL4-Sp3-N and GAL4-Sp3-B were created by PCR amplifying the Sp3 regions corresponding to amino acids $1-554$ and 183-554 respectively, using CMV-Sp3-Flu as a template and the following primers: Sp3-N, 5' CGGGATCCGTAATTCCGGGCCATCGCCGGGCGAC; Sp3-B, 5' CGGGATCCAAGTAGTTGCTAATGTGCCTCTTG; Sp3-3', CGGGATCCTCAAATGTGTTGCTTCTTTTTCCCAAG.

The PCR products were subsequently cloned into the Bam HI site of pGAL0. The junctions of all fusion proteins were sequenced to ensure the integrity of the fusion protein's reading frame.

\section{Cell culture and transfection}

Human HaCaT cells were grown in MEM supplemented with $10 \%$ FBS. Transient transfections were carried out with the standard DEAE-Dextran method and the luciferase activities measured $24 \mathrm{~h}$ after the addition of $100 \mathrm{pM}$ human TGF- $\beta 1$ as described (7).

\section{Gel shift assay}

Complementary oligonucleotides representing the SV40 Sp1 DNA binding site have the sequence $5^{\prime}$-CAT TCT CCG CCC CAT GGC-3' and were labeled with $\left[\gamma^{-32} \mathrm{P}\right] \mathrm{ATP}$ by T4 polynucleotide kinase. $\mathrm{HaCaT}$ cells were treated with human TGF- $\beta 1$ for either $0,4,8,12$ or $24 \mathrm{~h}$ and the respective nuclear extract prepared according to Dignam et al. (14). Total nuclear protein $(5 \mu \mathrm{g})$ was incubated for $10 \mathrm{~min}$ on ice with $1 \mu \mathrm{g}$ of poly(dI.dC) as well as the appropriate oligonucleotide competitors or antiserum, as indicated in the figures, in a $20 \mu \mathrm{l}$ reaction containing $20 \mathrm{mM}$ HEPES (pH 7.5), $5 \mathrm{mM} \mathrm{MgCl}_{2}, 60 \mathrm{mM} \mathrm{KCl}, 1 \mathrm{mM}$ DTT, $0.1 \%$ Triton X-100 and 6\% glycerol. The end-labeled probe (1 ng) was then added and the incubation continued for $20 \mathrm{~min}$ at $30^{\circ} \mathrm{C}$. The protein-DNA complexes were resolved on a $4 \%$ non-denaturing polyacrylamide gel. The gel was dried and exposed to X-ray film.
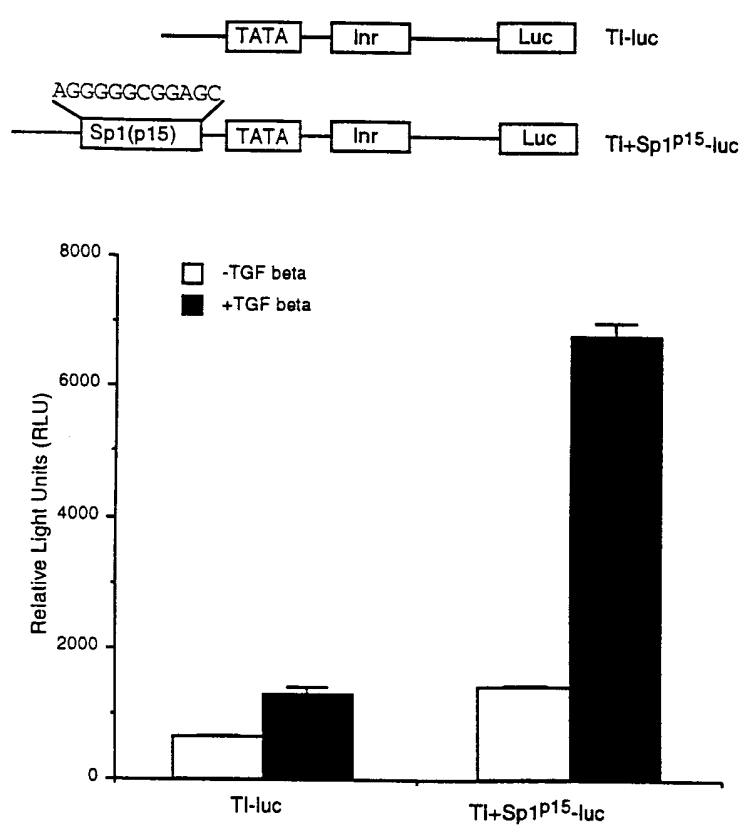

Induction Fold:

2

6

Figure 1. The essential $\mathrm{Sp} 1$ site present on the p15 promoter is sufficient for promoter induction by TGF- $\beta$ from a minimal luciferase reporter. Complementary oligonucleotides representing the essential $\mathrm{Sp} 1$ site present on the 15 promoter were inserted upstream of a minimal luciferase reporter, which contains the TATA and Inr elements in front of the luciferase reporter (TI-luc), to create TI+Sp1 $1{ }^{\mathrm{p} 15}$-luc. Both TI-luc and TI+Sp1 $1 \mathrm{P}^{\mathrm{15}}$-luc were transfected transiently into $\mathrm{HaCaT}$ cells and the relative luciferase activities assayed after cells were treated with TGF- $\beta$ for $24 \mathrm{~h}$. Induction folds by TGF- $\beta$ treatment are shown. Error bars represent the standard deviation of duplicates.

\section{RESULTS}

The essential Sp1 binding site required for TGF- $\beta$ induction of the p15 promoter is also sufficient for TGF- $\beta$ mediated promoter activation

Previously, we showed an Sp1 binding site present on the p15 promoter to be necessary for the induction of $\mathrm{p} 15$ promoter by TGF- $\beta$ treatment, since mutation of the Sp1 site abolished TGF- $\beta$ induction (7). To investigate whether the same Sp1 site is also sufficient for the promoter induction by TGF- $\beta$, we placed the $12 \mathrm{nt}$ $\mathrm{Sp} 1$ element upstream of a minimal luciferase reporter containing only the TATA and Inr elements (Fig. 1, TI-luc) to create TI+Sp1p15_luc. As shown in Figure 1, insertion of the p15 Sp1 element alone rendered the minimal luciferase reporter to become inducible by TGF- $\beta$ with more than six folds (Fig. 1, $\left.\mathrm{TI}+\mathrm{Sp} 1^{\mathrm{p} 15}-\mathrm{luc}\right)$. We routinely observed some residual induction of the minimal luciferase reporter by TGF- $\beta$ treatment (Fig. 1, TI). Thus, the same Sp1 binding site required for the TGF- $\beta$ induction of the $\mathrm{p} 15$ promoter is sufficient for TGF- $\beta$ mediated promoter activation when introduced into an exogenous minimal promoter. This result is consistent with our previous findings that the p21 promoter TGF- $\beta$ responsive element containing Sp1 binding site was also sufficient to confer TGF- $\beta$ responsiveness onto a heterologous promoter (8). 
A

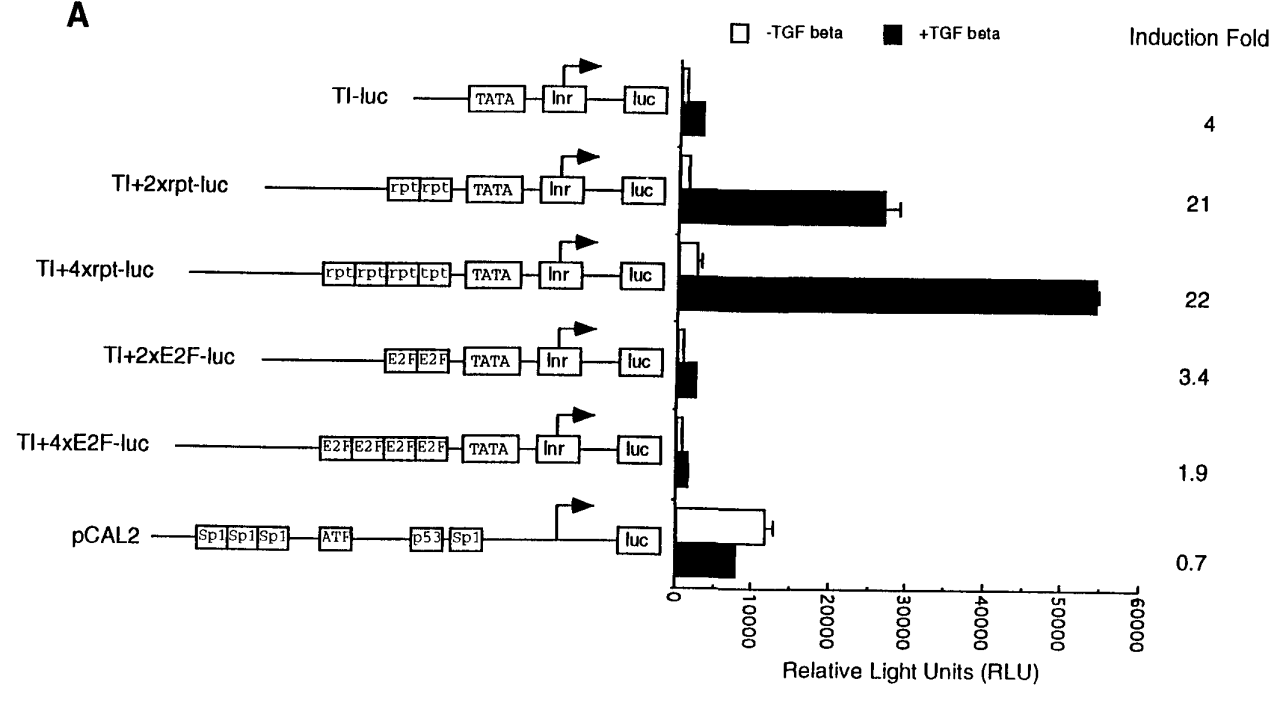

B

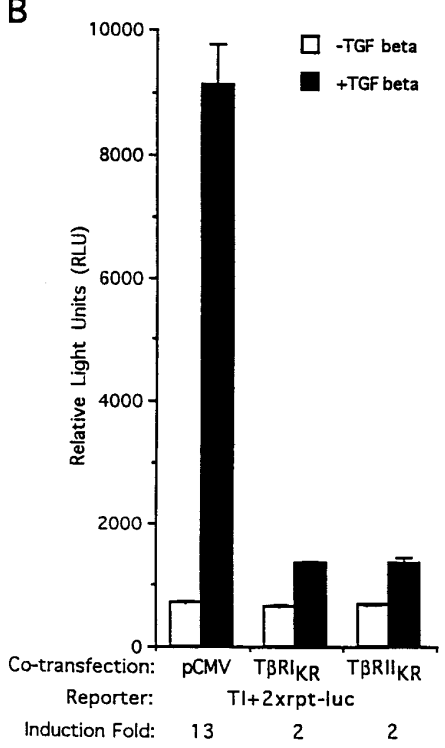

Figure 2. Canonical Sp1 binding sites from the SV40 21 repeats support TGF- $\beta$ mediated promoter activation. (A) Two or four copies of the SV40 21 repeat were

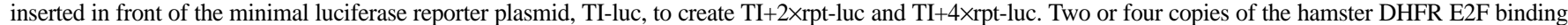
sites were introduced similarly to create TI $2 \times \mathrm{E} 2 \mathrm{~F}-\mathrm{luc}$ and $\mathrm{TI}+4 \times \mathrm{E} 2 \mathrm{~F}-\mathrm{luc}$. These constructs were transfected transiently into HaCaT cells and the relative luciferase activities assayed after cells were treated with TGF- $\beta$ for $24 \mathrm{~h}$. Induction folds by TGF- $\beta$ treatment are indicated. Error bars represent the standard deviation of duplicates. (B) TI $+2 \times$ rpt-luc was co-transfected with pCMV vector plasmid, a dominant negative TGF- $\beta$ type I receptor (T $\beta R I_{K R}$ ), or a dominant negative TGF- $\beta$ type II receptor $(\mathrm{T} \beta \mathrm{RII} / \mathrm{KR})$ as described in the text. Induction folds by TGF- $\beta$ treatment are indicated. Error bars represent the standard deviation of duplicates.

\section{Canonical Sp1 binding sites from the SV40 21 repeat support TGF- $\beta$ mediated gene induction}

The $\mathrm{Sp} 1$ sites from both the $\mathrm{p} 15$ and $\mathrm{p} 21$ promoters have been shown to bind to the $\mathrm{Sp} 1$ family of transcription factors, $\mathrm{Sp} 1, \mathrm{Sp} 3$ and $\Delta \mathrm{Sp} 3$, and thus represent true $\mathrm{Sp} 1$ binding sites $(7,8)$. To investigate if a canonical Sp1 binding site could also support TGF- $\beta$ mediated gene activation, we generated luciferase reporter constructs driven by either two or four copies of the 21 repeats from the SV40 early enhancer, in addition to the TATA and Inr elements (Fig. 2A). SV40 early enhancer contains three copies of the so-called 21 repeats to which $\mathrm{Sp} 1$ factor was originally shown to bind in a sequence specific manner (15).

These constructs were transfected transiently into HaCaT cells and the luciferase activities assayed after cells were treated with or without TGF- $\beta$ for $24 \mathrm{~h}$. As shown in Figure $2 \mathrm{~A}$, TGF- $\beta$ treatment caused a $>20$-fold increase of luciferase activity from

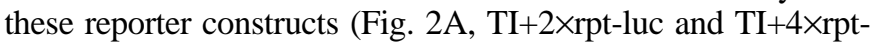
luc). On the contrary, concatemerization of E2F binding sites did not increase TGF- $\beta$ mediated gene expression. Instead, insertion of E2F binding sites consistently decreased the residual, induced expression from the minimal promoter (Fig. $2 \mathrm{~A}$, TI $+2 \times \mathrm{E} 2 \mathrm{~F}-\mathrm{luc}$ and $\mathrm{TI}+4 \times \mathrm{E} 2 \mathrm{~F}-\mathrm{luc})$, consistent with our earlier findings that $\mathrm{E} 2 \mathrm{~F}$ binding sites function to repress gene expression mediated by TGF- $\beta$ (16). TGF- $\beta$ mediated promoter induction from the canonical Sp1 sites depended on both the functional type I and type II TGF- $\beta$ receptors because a dominant negative (i.e. kinase deficient) type I TGF- $\beta$ receptor $\left(\mathrm{T} \beta \mathrm{RI}_{\mathrm{KR}}\right)$ or a dominant negative type II TGF- $\beta$ receptor $\left(\mathrm{T} \beta \mathrm{R} \mathrm{II}_{\mathrm{KR}}\right)$ specifically abolished TGF- $\beta$ mediated gene expression without affecting the uninduced expression (Fig. 2B). Thus, canonical Sp1 binding site could support TGF- $\beta$ mediated gene induction and therefore represents a distinct TGF- $\beta$ responsive element.

\section{TGF- $\beta$ does not change the DNA binding activities of Sp1, $\mathrm{Sp3}$ and $\Delta \mathrm{Sp3}$ transcription factors}

As shown in Figure 3A, three specific protein complexes were found to bind to the SV40 Sp1 site. They were readily competed by a 20 -fold excess of the unlabeled homologous SV40 Sp1 oligonucleotides (lane 2), but not by the same fold excess of the Gal4 or E2F binding oligonucleotides (lanes 4 and 5). All three specific complexes were also readily competed by a 20 -fold excess of the essential Sp1 binding site from the 15 promoter (lane 3), indicating that Sp1 sites from both the SV40 21 repeat and $\mathrm{p} 15$ promoter may bind to the same factors in $\mathrm{HaCaT}$ cells. Addition of antibodies against $\mathrm{Sp} 1$ in the gel retardation reaction specifically abolished the slowest migrating complex and caused formation of the supershifted complexes, indicating that this complex contained Sp1 factor (lane 6). Similarly, addition of specific antibodies against $\mathrm{Sp} 3$ in the gel retardation assay abolished the second- and third-slowest migrating complexes, indicating that they contained $\mathrm{Sp} 3$ and $\Delta \mathrm{Sp} 3$, respectively (lane 7 ). These results are consistent with our previous findings $(7,8)$ and further suggest that the Sp1 factors may mediate the TGF- $\beta$ induced promoter activation.

We next investigated if the DNA binding activities of Sp1, Sp3 or $\Delta \mathrm{Sp} 3$ were regulated by TGF- $\beta$. Nuclear extracts were prepared from $\mathrm{HaCaT}$ cells treated with TGF- $\beta$ for either $0,4,8,12$ or $24 \mathrm{~h}$ and used in a gel retardation assay. As shown in Figure 3B, TGF- $\beta$ treatment did not change DNA binding activities of either Sp1, $\mathrm{Sp} 3$ or $\Delta \mathrm{Sp} 3$ (Fig. 3B, lanes 1-5), suggesting that a mechanism other than altering the DNA binding activities of Sp1 transcription factors is responsible for the TGF- $\beta$-mediated transcriptional activation. This notion is further supported by our finding that the in vivo occupancy status of Sp1 sites of the p21 promoter in HaCaT cells 

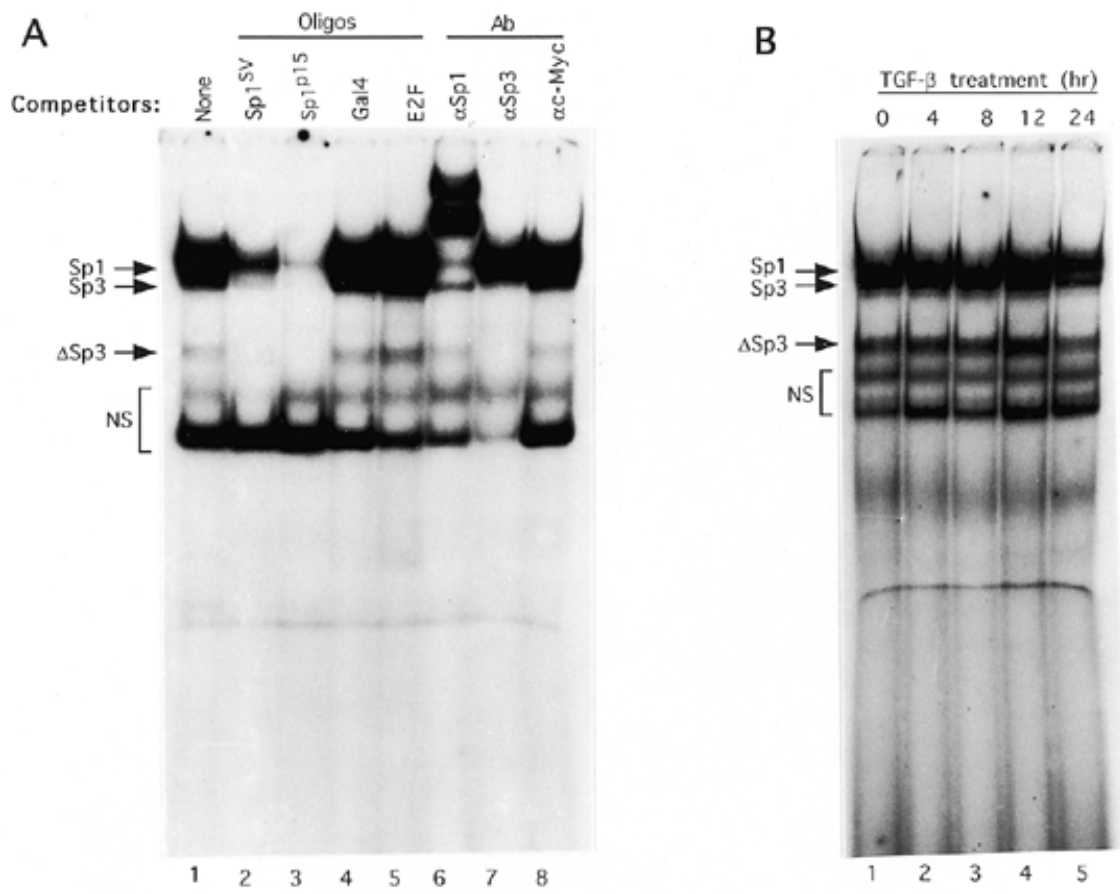

Figure 3. TGF- $\beta$ does not change DNA binding activities of Sp1 factors. (A) Complementary oligonucleotides representing the Sp1 binding site from the SV40 21 repeat were labeled with $\left[\gamma_{-}{ }^{32} \mathrm{P}\right] \mathrm{ATP}$ and used as a probe in the gel retardation assay with nuclear extract prepared from untreated HaCaT cells. Oligonucleotides or antibodies were included in the gel retardation reaction as specific competitors as indicated above each lane: lane 1, no competitor; lane 2, homologous SV40 Sp1 oligonucleotides; lane 3, oligonucleotides representing the essential Sp1 site of the p15 promoter; lane 4, oligonucleotides representing Gal4 binding site; lane 5, oligonucleotides representing the DHFR E2F binding site; lane 6, polyclonal antibodies against human Sp1; lane 7, polyclonal antibodies against human Sp3; lane 8, polyclonal antibodies against human c-Myc. Three specific complexes are indicated by arrows: Sp1, Sp3 and $\Delta \mathrm{Sp} 3$. Also indicated are the non-specific complexes (NS). (B) Nuclear extracts were prepared from HaCaT cells treated with TGF- $\beta$ for $0,4,8,12$ or 24 h and used in the gel retardation assay. Specific complexes are indicated as well as non-specific complexes (NS).

does not change with the treatment of TGF- $\beta$ (Datto and Wang, unpublished results).

\section{The B-subdomain of Sp1, but not Sp3, can mediate the responsiveness to TGF- $\beta$}

To test if Sp1 and/or Sp3 act as mediators of the TGF- $\beta$ transactivating signal, we used a system in which fragments of the transactivation domain of either $\mathrm{Sp} 1$ or $\mathrm{Sp} 3$ were fused to the DNA binding domain of the bacterial transcription factor GAL4. Consequently, the ability of these fusion proteins to activate transcription from a consensus GAL4 DNA binding site in both the presence and absence of TGF- $\beta$ could be measured. In this way, an enhancement of the transactivating capability of either $\mathrm{Sp} 1$ or $\mathrm{Sp} 3$ in response to TGF- $\beta$ should result in an augmented transcription from the GAL4 DNA binding site when an appropriate $\mathrm{Sp} 1$ or $\mathrm{Sp} 3 \mathrm{GAL} 4$ fusion protein is expressed.

As shown in Figure 4A, the transactivation domain of Sp1 can be divided into A and B subdomains $(15,17,18)$. Both of these subdomains contain glutamine rich motifs which are able to sufficiently activate transcription (18). The set of GAL4 DNA binding domain-Sp1 transactivation domain fusion proteins used in these experiments, which contain either the A-domain, the B-domain or fragments of the A- or B-domains, are depicted schematically in Figure 4A. To determine if the fusion proteins could still bind to DNA, we first performed gel shift analysis using nuclear extracts derived from COS cells overexpressing these proteins and demonstrated that all the fusion proteins retain the same capability in binding to the specific GAL4 binding sequences (data not shown). These GAL4 fusion expression constructs were next co-transfected into $\mathrm{HaCaT}$ cells with a luciferase reporter construct whose expression is driven by five consensus GAL4 DNA binding sites (Fig. 4A). Neither the reporter vector alone nor the reporter vector co-transfected with the vector expressing only the GAL4 binding domain were activated by TGF- $\beta$ treatment (Fig. 4B). When the $5 \times$ GAL4 reporter vector was co-transfected with a positive control plasmid expressing a fusion protein between the GAL4 DNA binding domain and the acidic activation domain of the viral transcription factor VP-16, a very high level of transcription is observed with no significant change upon TGF- $\beta$ treatment. In contrast to these results, expression of fusion proteins between the GAL4 DNA binding domain and the B-domain of Sp1 renders the $5 \times$ GAL 4 reporter construct TGF- $\beta$ responsive (Fig. 4B). This effect is also seen with smaller fragments of the B-domain which contain the glutamine-rich activation sequence. Thus, the B-domain of Sp1 can be activated by TGF- $\beta$ treatment to increase transcription. This result is in contrast to that seen for the GAL4-Sp1-A fusion proteins. Although the A-domain supported an increased level of basal transcription, neither the A-domain nor the glutamine-rich sequences of the A-domain was capable of activating transcription in response to TGF- $\beta$ (Fig. 4B). This suggests that the transactivating activity of Sp1 B-domain can be regulated in a specific fashion in response to an external signal, perhaps through phosphorylation or specific protein-protein 

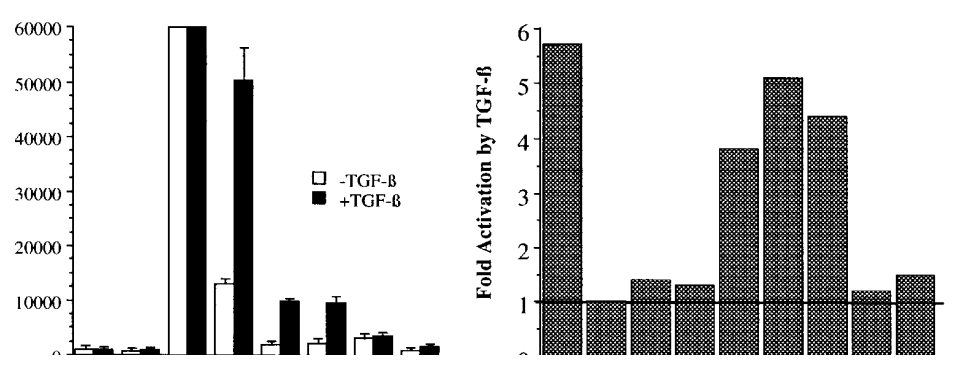

Figure 4. GAL4-Sp1, but not GAL4-Sp3, supports TGF- $\beta$ mediated promoter induction from GAL4 binding sites. (A) Schematically diagrammed reporter and the GAL4-Sp1 fusion protein constructs. The reporter construct pGL2 T+I 5XGAL4 contains five consensus GAL4 binding sites, a TATA box, and an initiator sequence driving the expression of a luciferase reporter gene. The domain structure of Sp1 is shown to indicate the A and B transactivation domains with their glutamine-rich and serine/threonine-rich motifs. The construct pGal0 expresses only the GAL4 DNA binding domain. The GAL4-Sp1 fusion expression constructs are shown to contain different regions of the A-domain or the B-domain of Sp1. (B) The B-domain of Sp1 can be activated by TGF- $\beta$. The results presented are the average of duplicate transfections in a single experiment. Error bars represent the standard deviation. The full length p21 promoter reporter construct p21P was included as a positive control for TGF- $\beta$ mediated transcriptional activation through a consensus Sp1 site. (C) Signaling from the type I and type II TGF- $\beta$ receptors is required for the activation of Sp1 B-domain by TGF- $\beta$. (D) Schematically diagrammed GAL4-Sp3 fusion protein constructs. The GAL4-Sp3 fusion expression constructs are shown to contain different regions of Sp3. (E) The transactivation domain of $\mathrm{Sp} 3$ cannot be activated by TGF- $\beta$. The same procedure was used as that for the GAL4-Sp1 fusion proteins. 